\title{
MAXILLARY SINUS SCHWANNOMA EXTENDING TO ORBIT
}

\author{
Sharma R*, Singh A K*, Shambhu B*, Reddy N S*
}

\section{ABSTRACT}

Schwannoma occur in the head and neck region more frequently than in any other part of the body, affecting mainly the eighth cranial nerve. However schwannoma in the nasal cavity and paranasal sinuses is a rare entity with only sporadic cases appearing in the literature. We present one such rare case of maxillary sinus schwannoma extending to nasal cavity orbit and anterior skull base in a 46 year old male. The tumor was successfully removed by midfacial degloving approach. The relevant literature on the presentation, radiology and management of schwannoma of maxillary sinus is discussed.

Key Words: Schwannoma, Maxillary Sinus, Nasal Cavity.

\section{INTRODUCTION}

Schwannoma are benign tumors arising from schwann cells that surround the axon of cranial nerves as they leave the central nervous system. They account for $8 \%$ of all intracranial tumors and most frequently arise from the vestibular division of vestibulocochlear nerve and less frequently from other cranial nerves.

They occur with the highest frequency in the head and neck region yet only $4 \%$ have been found to occur within the paranasal sinuses. ${ }^{1}$ Higo et $\mathrm{al}^{2}$ reviewed the medical literature and found 160 cases of schwannoma of nose and paranasal sinuses with only a few cases with intracranial extension. We present one such rare case encountered at our centre with a review of literature.

\section{CASE REPORT}

The patient, a 46 year old male presented to us with the history of off and on bleeding from right nostril for last one and a half year, gradually progressive nasal obstruction for last six months and bulging of right eye for last 3 months. There was no history of anosmia, unconciousness, visual impairment, seizures, headache and vomiting.

General and systemic examination was normal. Local examination showed and a greyish white firm mass in right nostril upto the level of middle turbinate and pushing the nasal septum to left side. On probing there was no bleeding. Ophthalmic evaluation showed proptosis of right eye, bilateral $6 / 6$ vision, no field defect and normal fundus. There was no significant cervical lymphadenopathy.

* Manipal College of Medical Sciences and Manipal Teaching Hospital, Pokhara, Nepal.

Address for correspondence : Dr. Ravinder Sharma

Dept. of ENT

Manipal College of Medical Sciences \& Manipal Teaching Hospital

Phulbari, Post Box: No. 341, Pokhara, Nepal

Email: rsharma45@yahoo.com 
CT scan showed a large enhancing mass lesion in right maxillary sinus, nasal cavity, ethmoids and extending to orbit and anterior skull base (Figure 1 A-B). There was no

Fig. 1 (A)

Fig.1(A) : Coronal CT scan showing mass lesion in the maxillary sinus and the nasal cavity extending to orbit and anterior skull base.

intracranial extension. On the basis of clinical evaluation and
CT scan the relatively common conditions of this region e.g. esthesioneuroblastoma, fungal granuloma and nasoethmoid carcinoma were considered in the differential diagnosis. A biopsy was taken under local anaesthesia using zero degree nasal endoscope. It was reported as schwannoma.

Patient was planned for surgery by midfacial degloving approach in view of the extension of tumour in orbit $\&$ anterior skull base. Incision was given in the upper gingivo labial sulcus (from right last molar to left last molar) and circumvestibular incisions were made in both the nostril. Dissection was done to combine gingivolabial and circumvestibular incisions and to elevate the skin of mid one third of face-from gingivolabial sulcus to the root of the nose. This provides a good exposure of anterior skull base. After elevating flap, a window was made in anterolateral wall of right maxillary sinus. It was widened to expose the greyish white, firm vascular mass in the maxillary sinus \& nasal cavity. The medial wall of right maxillary sinus was thinned out with erosion in the region of middle turbinate. The tumor was removed in parts and the medial wall of right maxillary sinus removed. There was a small defect in the roof of maxillary sinus. It was widened and a small extension in orbit was removed. After complete removal nasal cavity was packed with antibiotic medicated pack and closure was done with $3 / 0$ vikryl.

Light microscopy of the excised mass (Figure 2) showed

Fig. 2

Fig.2 : High power photomicrograph showing fascicles of spindle shaped cells with nuclear regimentation.

fragmented bits of tumor composed of tightly packed and irregularly running fascicles of spindle shaped cells with delicate slender nuclei (Antoni A tissue), nuclear regimentation in places and fibrillary eosinophilic cytoplasm associated with hyalinized stroma containing many dilated blood vessels.

Post operative period was uneventful and pack was removed after 48 hours. The patient was discharged on fifth postoperative day in satisfactory condition. At three months, six months \& one year follow up the patient was asymptomatic and on clinical examination. 
There was no evidence of recurrence or residual tumor. CT scan at 3 months follow up showed operated cavity and no evidence of residual tumor (Figure 3).

Fig. 3

Fig. 3 : Post operative coronal CT scan showing the operated cavity. There was no evidence of residual tumor in the nasal cavity and maxillary sinus.

\section{DISCUSSION}

Schwannoma are common tumors arising from neural sheath of peripheral nerves, autonomic nerves or cranial nerves. Rarely they involve nose and paranasal sinuses. ${ }^{2}$ The lesion most commonly arises in the ethmoid and maxillary sinus, ${ }^{3}$ rarely they arise from nasal tip, ${ }^{4}$ nasal septum ${ }^{5}$ and palate. ${ }^{6}$

Schwannoma of nasoethmoidal complex can occur at any age but the peak incidence is in second to fourth decade. ${ }^{7}$ Trimas et al ${ }^{8}$ suggested that in AIDS patients nerve sheath tumors must be included in the differential diagnosis of sinus masses. They reported one such case. The patient in our case had recurrent bleeding from right nostril for last one and a half year suggesting a slowly growing tumor unlike esthesioneuroblastoma, nasoethmoid carcinoma, more common lesions of region which usually have such history of short duration.

They are slowly progressive and rarely extends intracranially in the anterior cranial fossa ${ }^{7,9}$ or middle cranial fossa. ${ }^{10}$ They are usually discovered late because of lack of early symptoms ${ }^{11}$ and complex anatomy of this region.

CT scan helps in the assessment of tumor extension and in deciding the surgical approach. In our case, determination that this mass lesion was schwannoma was difficult on the basis of CT scan as schwannoma do not have a specific radiological finding. The tumor gradually extends to the skull base and intracranially due to bony erosion. Radiologically it may look malignant but histopathology of the resected specimen helps in the diagnosis. Fujiyoshi et $\mathrm{al}^{12}$ suggested that MR imaging clearly delineate the anatomic extension of the tumor and CT offered better resolution than MR imaging of the remodeled or interrupted bone of the skull base. He suggested that CT and MRI both are useful for determining the location and extension of the tumor. MRI was not done in our case

In our case endoscopic nasal biopsy was taken and it was reported as schwannoma. Klossek et al $^{11}$ also advocated the role of endoscopy in the diagnosis of sinunasal schwannoma. Butagen et $\mathrm{al}^{5}$ diagnosed two cases of schwannoma of nasal septum on the basis of immunohistochemical studies as nasal biopsy was inconclusive in these cases. In our case, the origin of the tumor is obscure and the tumor presumably arose in the maxillary sinus and then extended to nasal cavity, ethmoids, orbit and skull base. However the nerve from which tumor arose is uncertain. Bavetta et $\mathrm{al}^{7}$ suggested anterior ethmoidal nerve or meningeal branch of trigeminal nerve as the nerve of origin of this tumor.

The treatment of choice in schwannoma is surgical excision of the tumor. ${ }^{9}$ The selection of approach depends on the anatomic extension of tumor. Midfacial degloving is known to provide excellent exposure of the anterior and middle skull base without facial incision. The patient with extension in orbit and anterior skull base was operated by midfacial degloving approach. Younis et $\mathrm{al}^{13}$ suggested postoperative external radiotherapy and radon seed implants for such patients.

\section{ACKNOWLEDGEMENT}

The author wishes to thank Professor (Dr.) J. S. Nagra Dean \& Director Manipal College of Medical Sciences Pokhara for giving permission to publish this article.

\section{REFERENCES}

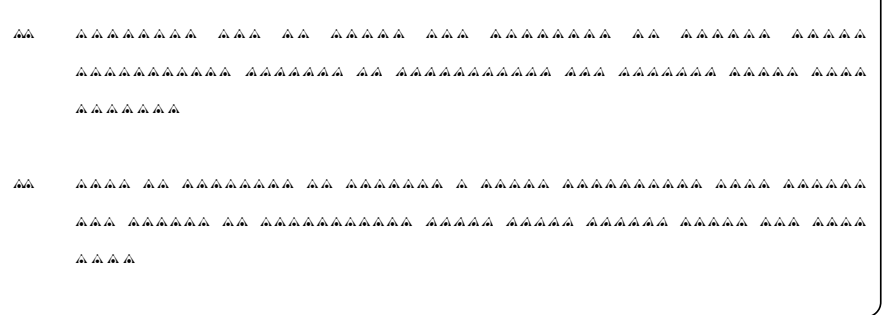




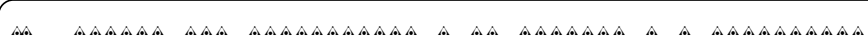

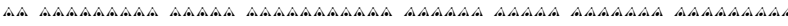

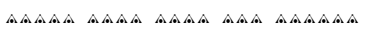

s..

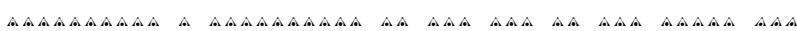

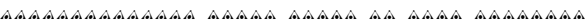

s.s.

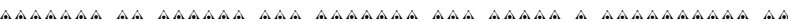

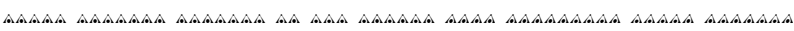

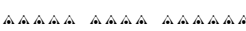

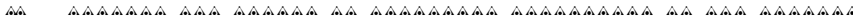

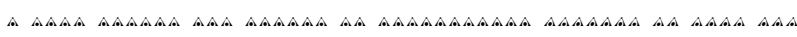

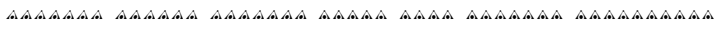

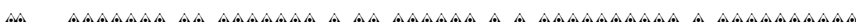

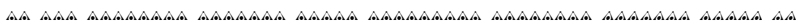

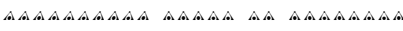

is.

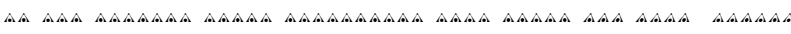
$\triangle A \triangle A \triangle A A \triangle A \triangle A \triangle A \triangle A \triangle A \triangle A \triangle A A A$
}

As.

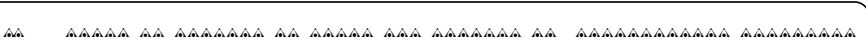

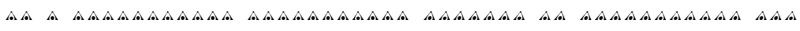
$\triangle A \triangle A \triangle A A$ AAAAA AAAA AAA A AAA

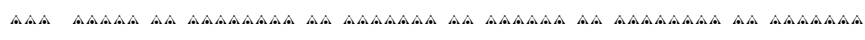

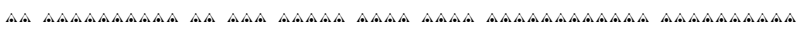

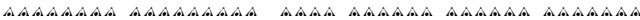

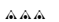

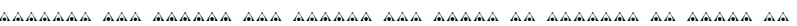

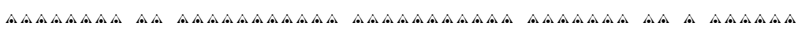

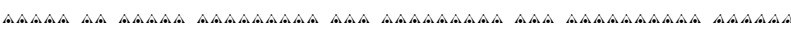

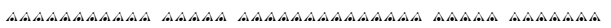

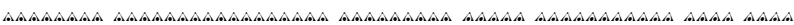
$\triangle A \triangle A \triangle A \triangle A \triangle A \triangle A \triangle A \triangle A \triangle A \triangle A \triangle A \triangle A$

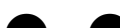

